\title{
A COMPLEMENT TO THE CONNECTING LEMMA OF HAYASHI
}

\author{
JOSÉ CARLOS MARTÍN† AND LEONARDO MORA \\ To J. Palis on his 60th anniversary.
}

\begin{abstract}
Here we show that for a $\mathrm{C}^{2}$ surface diffeomorphism that satisfy the hypothesis of Hayashi connecting lemma either can be approximated, in the $\mathrm{C}^{1}$ topology, by a diffeomorphism exhibiting a homoclinic tangency or the diffeomorphism already presented transversal homoclinic orbits.
\end{abstract}

\section{INTRODUCTION}

Let $M$ be a $\mathrm{C}^{\infty}$ compact surface and $f: M \rightarrow M$ be a $\mathrm{C}^{1}$ diffeomorphism. Let $\Lambda$ be an isolated hyperbolic set, we shall say that $q$ is a homoclinic point (of $f$ ) associated to $\Lambda$ if $q \in W^{s}(\Lambda, f) \cap W^{u}(\Lambda, f) \backslash \Lambda$, where

$$
\begin{gathered}
W^{s}(X, f)=\left\{x \in M: \lim _{n \rightarrow \infty} \operatorname{dist}\left(f^{n}(x), f^{n}(X)\right)=0\right\} \\
\left(\text { resp. } W^{u}(X, f)=\left\{x \in M: \lim _{n \rightarrow-\infty} \operatorname{dist}\left(f^{n}(x), f^{n}(X)\right)=0\right\}\right)
\end{gathered}
$$

is the stable (resp. unstable) set (manifold) of $X$. When $f$ is well understood from context we shall put simply $W^{s}(X)$ and $W^{u}(X)$. Let $p$ be a hyperbolic periodic point of $f$ and $q$ a homoclinic point associated to $p$, we shall say that $q$ is a transversal homoclinic point if $T_{q} W^{s}(p)+T_{q} W^{u}(p)=T_{q} M$, in the opposite case we shall say that $q$ is a homoclinic tangency associated to $p$. A sequence $\left\{\gamma_{n}\right\}$ of finite orbits of $f$ is called an almost homoclinic sequence associated to $\Lambda$ if there exist $U$, a neighborhood of $\Lambda$, and $\epsilon>0$ such that:

i. $\bigcap_{j \in \mathbb{Z}} f^{j}(U)=\Lambda$,

ii. there exist $q^{s} \in W_{\epsilon}^{s}(\Lambda) \backslash \Lambda, q^{u} \in W_{\epsilon}^{u}(\Lambda) \backslash \Lambda, q_{n}^{\prime} \in \gamma_{n}$ and $m_{n}^{\prime} \in \mathbb{Z}^{+}$, for all $n \in \mathbb{Z}^{+}$, such that $q^{u}=\lim _{n \rightarrow \infty} q_{n}^{\prime}$ and $q^{s}=\lim _{n \rightarrow \infty} f^{m_{n}^{\prime}}\left(q_{n}^{\prime}\right)$, iii. $\left\{q_{n}^{\prime}, f\left(q_{n}^{\prime}\right), \ldots, f^{m_{n}^{\prime}}\left(q_{n}^{\prime}\right)\right\} \cap(M \backslash U) \neq \emptyset$, for all $n \in \mathbb{Z}^{+}$, where $W_{\epsilon}^{s}(\Lambda)=\bigcup_{x \in \Lambda} W_{\epsilon}^{s}(x)$ and $W_{\epsilon}^{u}(\Lambda)=\bigcup_{x \in \Lambda} W_{\epsilon}^{u}(x)$ are the local stable and unstable manifolds of $\Lambda$ respectively. We shall say that an invariant set $\Lambda$ of $f$ is a basic set if it is a compact, hyperbolic, isolated and transitive.

The $\mathrm{C}^{1}$ connecting lemma of Hayashi [H] says that, if a compact isolated hyperbolic set $\Lambda$ has associated an almost homoclinic sequence, then for all $\mathrm{C}^{1}$ neighborhood $\mathcal{U}$ of $f$, there exists $g \in \mathcal{U}$ coinciding with $f$ in a neighborhood of $\Lambda$ and having a homoclinic point associated to a periodic hyperbolic point in $\Lambda$. Our purpose here is to show the following theorem.

Date: 7th November 2018.

†Work partially supported by IMPA-Brasil.

‡Work partially supported by IMPA-Brasil, CONICIT-Venezuela and Fundación Polar. 
Theorem 1. If $f: M^{2} \rightarrow M^{2}$ is a $\mathrm{C}^{2}$ diffeomorphism with a basic set $\Lambda$ which has associated an almost homoclinic sequence then for every periodic point $p \in \Lambda$ one of the following statements hold:

(i) $p$ has associated a transversal homoclinic point outside $\Lambda$,

(ii) for all $\mathcal{N} \subset \operatorname{Diff}^{1}(M)$, a neighborhood of $f$, there exists $g \in \mathcal{N}$ having a homoclinic tangency associated to $p$.

In $|\mathrm{PS}|$, it is proved that given a diffeomorphism $f: M^{2} \rightarrow M^{2}$, it can be $\mathrm{C}^{1}$ approximated by a diffeomorphism exhibiting a homoclinic tangency or by an Axiom A diffeomorphism. Since the dynamical richness exhibited by nearby diffeomorphisms of another one exhibiting a homoclinic tangency [PT], it is an important problem to decide when, in a particular dynamical situation, the system can be approximated by another one having a homoclinic tangency. We raise this question in the following dynamical situation: Suppose that $f: M^{2} \rightarrow M^{2}$ is a $\mathrm{C}^{2}$ diffeomorphism which has a compact basic set $\Lambda$ and $q \in\left(\left(\overline{W^{s}(\Lambda)} \cap W^{u}(\Lambda)\right) \cup\left(\overline{W^{u}(\Lambda)} \cap W^{s}(\Lambda)\right)\right) \backslash \Lambda$. The Hayashi $\mathrm{C}^{1}$ connecting lemma says to us that in this case we can approximate $f$ by another diffeomorphism $g$ having a homoclinic orbit. But it does not say what kind of homoclinic orbit it has. The following corollary of theorem 1 give us the answer.

Corollary 1. Let $f: M^{2} \rightarrow M^{2}$ is a $\mathrm{C}^{2}$ diffeomorphism which has a basic set $\Lambda$ and $q \in\left(\left(\overline{W^{s}(\Lambda)} \cap W^{u}(\Lambda)\right) \cup\left(\overline{W^{u}(\Lambda)} \cap W^{s}(\Lambda)\right)\right) \backslash \Lambda$. If $W^{s}(\Lambda) \cap W^{u}(\Lambda) \backslash\{\Lambda\}=\emptyset$, then for every $\mathrm{C}^{1}$ neighborhood $\mathcal{N} \subset \operatorname{Diff}^{1}(M)$ of $f$ and for every hyperbolic periodic point $p \in \Lambda$, there exists $g \in \mathcal{N}$ having a homoclinic tangency associated to a $p$.

We do not know if the $\mathrm{C}^{2}$ hypothesis on $f$ above can be raised. The reason of its appearance is that we use results of [PS] which assume this hypothesis.

Respect to what happens in higher dimensions, we remark that examples of Díaz [D] make the theorem 1 false. What we think should be true is the following.

Conjecture 1. If $f: M \rightarrow M$ is a $\mathrm{C}^{2}$ diffeomorphism with a compact basic set $\Lambda$ which has associated an almost homoclinic sequence, then for every hyperbolic periodic point $p \in \Lambda$ one of the following statements hold:

(i) $p$ has associated a transversal homoclinic point outside $\Lambda$,

(ii) for all $\mathcal{N} \subset \operatorname{Diff}^{1}(M)$, a neighborhood of $f$, there exists $g \in \mathcal{N}$ having a homoclinic bifurcation associated to $p$.

Here we use the notion of homoclinic bifurcation as defined in [PT], page 134.

The paper is organized in three sections. In the second one we present all stuff which is necessary for doing the proof of the theorem, and in the last section we provide the proof of the theorem. The proof's idea is the following. Let $f$ and $\Lambda$ be as in the theorem. By the connecting lemma of Hayashi, $f$ can be approximated by diffeomorphisms exhibiting a transversal homoclinic orbit. Analyzing the rate of domination between stable and unstable vectors for these homoclinic orbits, we obtain: If these rates are bounded for infinitely many perturbations, then $f$ has a homoclinic orbit outside $\Lambda$. In the other case $f$ can be approximated by diffeomorphisms exhibiting a homoclinic tangency.

\section{Preliminaries}

Consider a diffeomorphism $f: M \rightarrow M$ and a basic set $\Lambda$ of $f$ which has associated an almost homoclinic sequence $\left\{\gamma_{n}\right\}$. Let $q^{s}, q^{u}, q_{n}^{\prime}$ and $m_{n}^{\prime}$ as in definition 


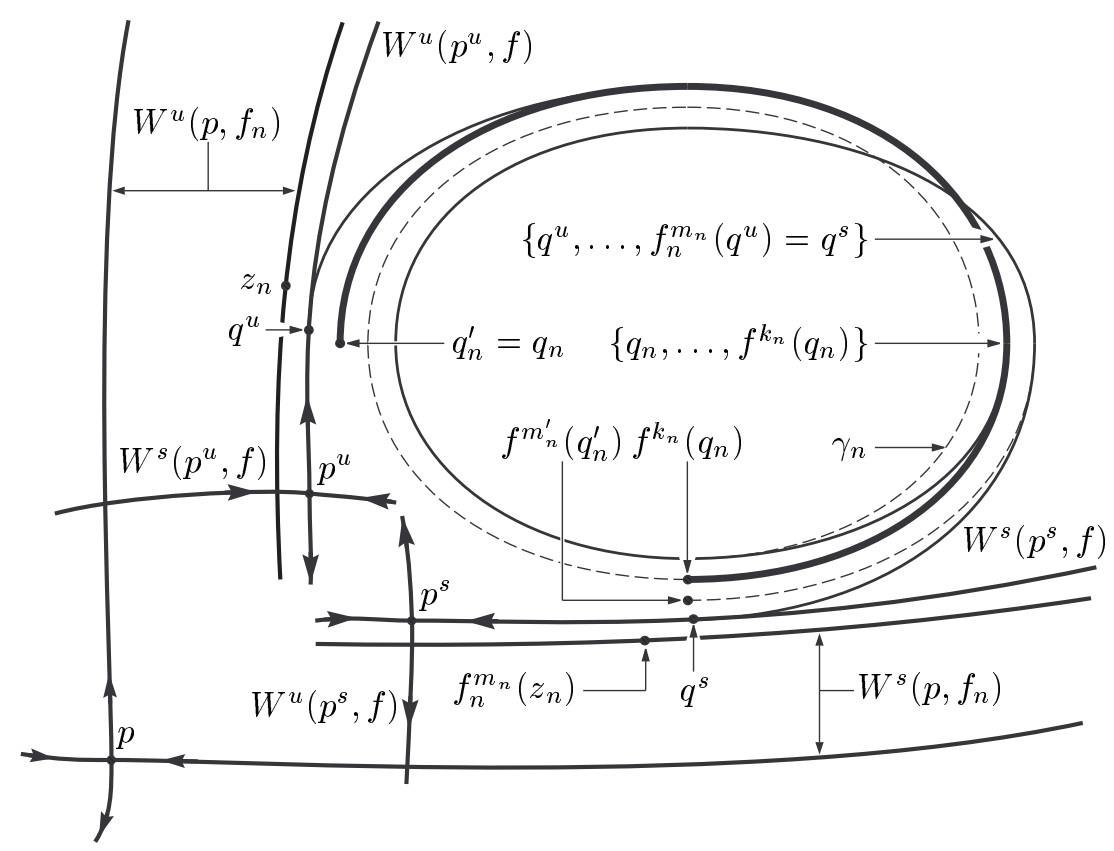

Figure 1. Proof of Lemma 1: Connection between stable and unstable manifolds of the orbits of $p^{s}$ and $p^{u}$, respectively. Compare with Figure $1(\mathrm{~B})$ of $[\mathrm{H}]$.

of an almost homoclinic sequence. Denote by $p^{s}$ and $p^{u}$ the points of $\Lambda$ such that $q^{s} \in W_{\mathrm{loc}}^{s}\left(p^{s}\right)$ and $q^{u} \in W_{\mathrm{loc}}^{u}\left(p^{u}\right)$. Let $p \in \Lambda$ be any hyperbolic periodic point.

Lemma 1. If $W^{s}(\Lambda) \cap W^{u}(\Lambda) \backslash \Lambda=\emptyset$ then there exist sequences $\left\{f_{n}\right\} \subset \operatorname{Diff}^{1}(M)$, $\left\{z_{n}\right\} \subset M,\left\{q_{n}\right\} \subset M,\left\{k_{n}\right\} \subset \mathbb{Z}^{+}$and $\left\{m_{n}\right\} \subset \mathbb{Z}^{+}$such that:

i. $f_{n} \rightarrow f$, in the $\mathrm{C}^{1}$ topology,

ii. for each $n, z_{n}$ is a transversal homoclinic point of $f_{n}$ associated to $p$,

iii. $\lim _{n \rightarrow \infty} z_{n}=q^{u}$ and $\lim _{n \rightarrow \infty} f_{n}^{m_{n}}\left(z_{n}\right)=q^{s}$;

iv. $\lim _{n \rightarrow \infty} q_{n}=q^{u}$ and $\lim _{n \rightarrow \infty} f^{k_{n}}\left(q_{n}\right)=q^{s}$, and

v. $\forall \epsilon>0$ there exists $N \in \mathbb{Z}^{+}$such that $f^{j}\left(q_{n}\right) \in \bigcup_{k=0}^{m_{n}} B_{\epsilon}\left(f_{n}^{k}\left(z_{n}\right)\right)$, for all $0 \leq j \leq k_{n}$ and $n \geq N$.

Remark 1. We observe that the hypothesis $W^{s}(\Lambda) \cap W^{u}(\Lambda) \backslash \Lambda=\emptyset$ is equivalent to $m_{n}^{\prime} \rightarrow \infty$ for all almost homoclinic sequences. Because if $m=\liminf _{n \rightarrow \infty} m_{n}^{\prime}<\infty$ then $f^{m}\left(q^{u}\right)=q^{s}$ that implies that $W^{s}(\Lambda) \cap W^{u}(\Lambda) \backslash \Lambda \neq \emptyset$. The converse is obvious.

For the proof of this lemma we use the following version of the connecting lemma.

Theorem 2. Let $M$ be a Riemannian manifold, $f: M \rightarrow M$ be a $\mathrm{C}^{1}$ diffeomorphism and $z$ be a non periodic point of $f$. Then for every neighborhood $\mathcal{U}$ of $f$ in $\operatorname{Diff}^{1}(M)$ there exist $\rho>1, L \in \mathbb{Z}^{+}$and $\delta_{0}>0$ such that for each $0<\delta \leq \delta_{0}$ and each couple of points, $x \notin T=\bigcup_{n=1}^{L} f^{-n} B_{\delta}(z)$ and $y \in B_{\delta / \rho}(z)$, for which the future orbit of $x$ mets $B_{\delta / \rho}(z)$, there exist $g \in \mathcal{U}$ and $m \in \mathbb{Z}^{+}$such that $g=f$ outside of $T$ and $g^{m}(x)=y$. 
This theorem is Theorem $\mathrm{F}$ of $\mathrm{WX}$.

Remark 2. In the conditions and notation of theorem 2. If $k \geq L$ is the first positive integer such that $f^{k}(x) \in B_{\delta}(z)$, then $k \leq m, f^{k}(x) \in B_{2 \delta}(y)$ and $f^{j}(x) \in$ $\bigcup_{n=0}^{m} B_{2 \delta}\left(g^{n}(x)\right)$, for all $0 \leq j \leq k$.

Proof of lemma 1. Because of the remark 1 , we know that $m_{n}^{\prime} \rightarrow \infty$. Take a sequence $\left\{\epsilon_{n}\right\}$ such that $\epsilon_{n} \rightarrow 0$. For each $n$ we take $\rho_{n}^{-}, L_{n}^{-}$and $\delta_{n}^{-}$as in Theorem 2 for $\mathcal{U}=B_{\epsilon_{n}}(f)=\left\{g \in \operatorname{Diff}^{1}(M): \operatorname{dist}_{\mathrm{C}^{1}}(g, f)<\epsilon_{n}\right\}$ and constants $\rho_{n}^{+}, L_{n}^{+}$and $\delta_{n}^{+}$ for $B_{\epsilon_{n}}\left(f^{-1}\right)$. Let $\rho_{n}=\max \left\{\rho_{n}^{-}, \rho_{n}^{+}\right\}, L_{n}=\max \left\{L_{n}^{-}, L_{n}^{+}\right\}$and $\delta_{n}=\min \left\{\delta_{n}^{-}, \delta_{n}^{+}\right\}$. Given $\delta>0$ we define $T_{n}^{-}(\delta)=\bigcup_{j=1}^{L_{n}} f^{-j}\left(B_{\delta}\left(q^{s}\right)\right)$ and $T_{n}^{+}(\delta)=\bigcup_{j=1}^{L_{n}} f^{j}\left(B_{\delta}\left(q^{u}\right)\right)$. It is easy to see there exist $0<\delta_{n}^{\prime} \leq \delta_{n}$ such that

i. $\left\{B_{\delta_{n}^{\prime}}\left(q^{u}\right), f\left(B_{\delta_{n}^{\prime}}\left(q^{u}\right)\right), \ldots, f^{2 L_{n}}\left(B_{\delta_{n}^{\prime}}\left(q^{u}\right)\right), B_{\delta_{n}^{\prime}}\left(q^{s}\right), \ldots, f^{-2 L_{n}}\left(B_{\delta_{n}^{\prime}}\left(q^{s}\right)\right)\right\}$ is a family of disjoint sets,

ii. $\overline{T_{n}^{ \pm}\left(\delta_{n}^{\prime}\right)} \cap \Lambda=\emptyset$,

iii. $T_{n}^{-}\left(\delta_{n}^{\prime}\right) \cap \bigcup_{j \geq 0} f^{-j}\left(W_{\mathrm{loc}}^{u}\left(p^{u}\right)\right)=\emptyset$ and $T_{n}^{+}\left(\delta_{n}^{\prime}\right) \cap \bigcup_{j \geq 0} f^{j}\left(W_{\mathrm{loc}}^{s}\left(p^{s}\right)\right)=\emptyset$.

In particular, $T_{n}^{-}\left(\delta_{n}^{\prime}\right) \cap T_{n}^{+}\left(\delta_{n}^{\prime}\right)=\emptyset$. Let $\left\{\alpha_{n}\right\} \subset \mathbb{Z}^{-}$such that $q_{\alpha_{n}}^{\prime} \in B_{\delta_{n}^{\prime} / \rho_{n}}\left(q^{u}\right)$ and $f^{m_{\alpha_{n}}^{\prime}}\left(q_{\alpha_{n}}^{\prime}\right) \in B_{\delta_{n}^{\prime} / \rho_{n}}\left(q^{s}\right)$, for easy of writing let us assume that $\alpha_{n}=n$, see figure 11. By theorem 2 there are a sequence of diffeomorphisms $\left\{g_{n}\right\}$ and a sequence of positive integers $\left\{m_{n}\right\}$ such that $g_{n}=f$ outside of $T_{n}^{-}\left(\delta_{n}^{\prime}\right), g_{n}^{m_{n}}\left(q_{n}^{\prime}\right)=$ $g_{n}^{m_{n}-L_{n}-1}\left(f^{L_{n}+1}\left(q_{n}^{\prime}\right)\right)=q^{s}$. In the same way, we get a sequence of diffeomorphisms $\left\{\tilde{g}_{n}\right\}$ such that $\tilde{g}_{n}=f$ outside of $T_{n}^{+}\left(\delta_{n}^{\prime}\right)$ and $\tilde{g}_{n}^{L_{n}+1}\left(q^{u}\right)=f^{L_{n}+1}\left(q_{n}^{\prime}\right)$.

Let $f_{n}: M \rightarrow M$ be defined by

$$
f_{n}(x)= \begin{cases}f(x) & \text { if } x \notin T_{n}^{-}\left(\delta_{n}^{\prime}\right) \cup T_{n}^{+}\left(\delta_{n}^{\prime}\right) \\ g_{n}(x) & \text { if } x \in T_{n}^{-}\left(\delta_{n}^{\prime}\right) \\ \tilde{g}_{n}(x) & \text { if } x \in T_{n}^{+}\left(\delta_{n}^{\prime}\right) .\end{cases}
$$

Because of the construction, $f_{n} \rightarrow f, \Lambda$ is a hyperbolic set of $f_{n}, f_{n}\left(q^{u}\right)=q^{s}$, $W_{\text {loc }}^{s}\left(p^{s}, f_{n}\right)=W_{\text {loc }}^{s}\left(p^{s}, f\right)$ and $W_{\text {loc }}^{u}\left(p^{u}, f_{n}\right)=W_{\text {loc }}^{u}\left(p^{u}, f\right)$. We can suppose that $W^{s}\left(p^{s}, f_{n}\right)$ and $W^{u}\left(f^{m_{n}}\left(p^{u}\right), f_{n}\right)$ meet in a transversal way at $q^{s}$, if not with a small perturbation we get that. Since $\bigcup_{j=0}^{k-1} W^{s}\left(f^{j}(p), f_{n}\right)$ and $\bigcup_{j=0}^{k-1} W^{u}\left(f^{j}(p), f_{n}\right)$ are dense in $\Lambda$, for all $n$, where $k \geq 1$ is the period of $p$, we have that $q^{u}$ is accumulated by transversal homoclinic points of $f_{n}$ associated to $p$, so $q^{u}$ is accumulated by transversal homoclinic points of $f_{n}$ associated to $p$. In particular we can take $z_{n}$, a homoclinic point of $f_{n}$ associated to $p$, in such a way that it holds (iii). The existence of $q_{n}$ and $k_{n}$ satisfying (iv) and (v) is an immediate consequence of the previous thing and of remark 2 .

The next lemma is the basic tool for making perturbations and is a slightly general version of [F, Lemma 1.1].

Lemma 2. Let $f \in \operatorname{Diff}^{1}(M)$. Given $\mathcal{U}, a \mathrm{C}^{1}$ neighborhood of $f$, there exist $\epsilon>0$ and a neighborhood $\mathcal{U}_{0} \subset \mathcal{U}$ of $f$ such that the following holds. If we have $\tilde{f} \in \mathcal{U}_{0}$, $\left\{x_{1}, \ldots, x_{n}\right\} \subset M, U$ a neighborhood of $\left\{x_{1}, \ldots, x_{n}\right\}$ and $T_{i}: T_{x_{i}} M \rightarrow T_{\tilde{f}\left(x_{i}\right)} M$ linear mappings with $\left\|T_{i}-D_{x_{i}} \tilde{f}\right\|<\epsilon$, for all $1 \leq i \leq n$, then there exists $g \in \mathcal{U}$ such that $g(x)=\tilde{f}(x)$, for $x \in\left\{x_{1}, \ldots, x_{n}\right\} \cup(M \backslash U)$, and $D_{x_{i}} g=T_{i}$, for all $1 \leq i \leq n$.

The following two lemmas are the tools to perturb $f$ along a piece of a homoclinic orbit to close the angle made by the stable and unstable manifolds. 
Lemma 3. For each $\delta>0$ and $\theta>0$ there exists $c>1$ such that the following holds. If $\left|b_{1}\right| /\left|b_{2}\right| \geq c$ and

$$
B=\left[\begin{array}{cc}
b_{1} & 0 \\
0 & b_{2}
\end{array}\right]
$$

then the angle between $e_{1}$ and $B \circ I_{\delta} e_{2}$ is less than $\theta$, where $I_{\delta}=\left[\begin{array}{ll}1 & \delta \\ 0 & 1\end{array}\right], e_{1}=\left(\begin{array}{l}1 \\ 0\end{array}\right)$ and $e_{2}=\left(\begin{array}{l}0 \\ 1\end{array}\right)$.

Proof. We may assume that $\theta<\frac{\pi}{2}$. If $c>\frac{1}{\delta \tan \theta}$, then $\angle\left(e_{1}, B \circ I_{\delta} e_{2}\right) \leq \angle\left(e_{1}, e_{1}+\right.$ $\left.c \delta e_{2}\right)<\theta$.

Now consider a sequence $\left\{A_{n}\right\}$ of diagonal matrices

$$
A_{n}=\left[\begin{array}{cc}
a_{n} & 0 \\
0 & b_{n}
\end{array}\right]
$$

with $a_{n}, b_{n}>0$. Let $\sigma_{n}=\prod_{j=0}^{n-1} a_{n}$ and $\tau_{n}=\prod_{j=0}^{n-1} b_{n}$.

Lemma 4. For each $\epsilon>0, \theta>0$ and $K>0$ there exists $r_{0} \in \mathbb{Z}^{+}$such that the following holds. If $\left\{A_{n}\right\}$ is a sequence of matrices as above with $\left\|A_{n}\right\| \leq K$ and $\sigma_{r} / \tau_{r}>1 / 2$, for $r \geq r_{0}$, then there exist matrices $\tilde{A}_{n}$, for $n=0, \ldots, r-1$, such that $\left\|\tilde{A}_{n}-A_{n}\right\|<\epsilon, \tilde{A}_{n} e_{1}=e_{1}$ and the angle between $e_{1}$ and $\tilde{A}_{r-1} \circ \cdots \circ \tilde{A}_{0} e_{2}$ is less than $\theta$.

Proof. First of all, we observe that there exists $\delta=\delta(\epsilon, K)$ such that if $C$ is a matrix $\delta$-close to the identity and $D$ is a matrix with $\|D\| \leq K$ then $\|C \circ D-D\|<\epsilon / 2$.

Let $c>1$ be given by Lemma 3 for $\delta$ and $\theta$, and let $r_{0} \in \mathbb{Z}^{+}$such that $(1+\delta)^{2 r_{0}} \geq$ 2c. If $\left\{A_{n}\right\}$ is a sequence as in the hypothesis and $r \geq r_{0}$, we define

$$
\hat{A}_{n}=\left[\begin{array}{cc}
(1+\delta) a_{n} & 0 \\
0 & \frac{1}{1+\delta} b_{n}
\end{array}\right], \quad \text { for } n=0, \ldots, r-1 .
$$

Clearly $\hat{A}_{n}$ is $\epsilon / 2$-close to $A_{n}$. In addition, $B=\hat{A}_{r-1} \circ \cdots \circ \hat{A}_{0}$ satisfies the hypothesis of Lemma 3 , so the angle between $e_{1}$ and $B \circ I_{\delta} e_{2}$ is less than $\theta$. Put $\tilde{A}_{n}=\hat{A}_{n}$, for $1 \leq n \leq r-1$, and $\tilde{A}_{0}=\hat{A}_{0} \circ I_{\delta}$. These matrices fulfill the thesis, so we are done.

Now we introduce some notation before presenting the next lemma. Let $x \in M$ be a fixed point of $f, \sigma$ and $\tau$ be the eigenvalues of $D_{x} f$ with $|\sigma|<|\tau|$. By the center stable manifold theorem, there exist invariant manifolds $W^{-}(x)$ and $W^{+}(x)$ associated to $\sigma$ and $\tau$ respectively. We shall say that $\Sigma$ is a separatrix of $W^{ \pm}(x)$ if $\Sigma$ is one of the connected component of $X \backslash\{x\}$, where $X$ is the connected component of $W^{ \pm}(x) \cap\left(W^{s}(x) \cup W^{u}(x)\right)$ which contains $x$. A separatrix $\Sigma$ will be called stable (resp. unstable) if $\Sigma \subset W^{s}(x)$ (resp. $\Sigma \subset W^{u}(x)$ ). When $x$ is a periodic point same definitions for separatrices extend considering $f^{k}$, where $k$ is the period of $x$. Observe that it can happen that a periodic point $x$ has not separatrices of $W^{ \pm}(x)$ at all. Denote by $\ell(\Sigma)$ the length of $\Sigma$.

Let $\Delta \subset M$ be an invariant compact set of $f$ and $E \oplus F=T_{\Delta} M$ be a $D f$ invariant splitting. We shall say that $E \oplus F$ is a dominated splitting of $\Delta$ if there exist $C>0$ and $0<\lambda<1$ such that

$$
\left\|\left.D f^{j}\right|_{E(x)}\right\|\left\|\left.D f^{-j}\right|_{F\left(f^{j}(x)\right)}\right\| \leq C \lambda^{j}
$$

for all $j \in \mathbb{Z}^{+}$and $x \in \Delta$. 


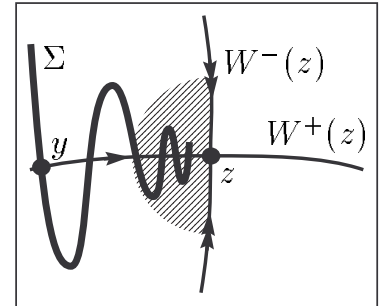

A

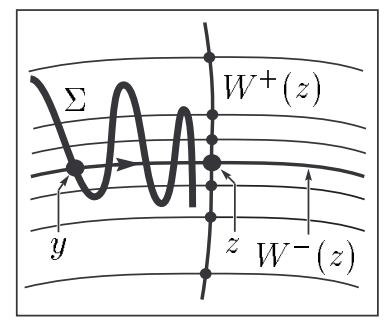

D

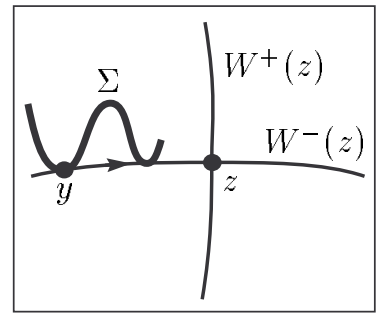

B

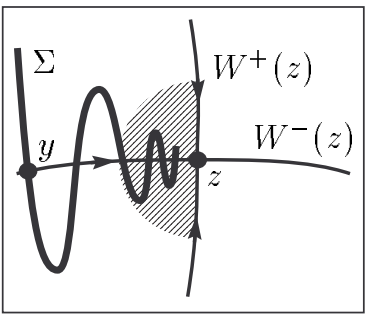

E
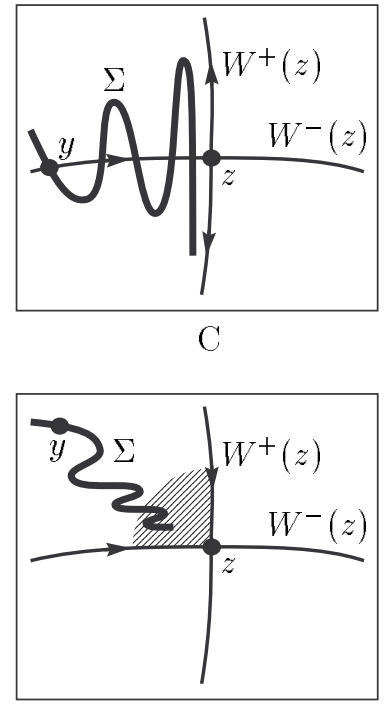

F

Figure 2. Proof of Lemma 5. The shadow parts correspond to a piece of the stable manifold of $z$.

Lemma 5. Let $\Delta$ be a compact invariant set with a dominated splitting $E \oplus F, x \in$ $\Delta$ be a periodic point, $\Sigma$ be an unstable (resp. stable) separatrix of $W^{+}(x)$ (resp. $W^{-}(x)$ ) and $y \in \Sigma \cap \Delta$. If $\ell(\Sigma)<\infty$ then there exist a neighborhood $U$ of $y$ and a periodic point $z$ such that $U \subset \bigcup_{n=1}^{k} W^{s}\left(f^{n}(z)\right)$ (resp. $U \subset \bigcup_{n=1}^{k} W^{u}\left(f^{n}(z)\right)$ ), where $k$ is the period of $z$.

Proof. We assume that $x$ is a fixed point, if $x$ is a periodic point take $f^{k}$, where $k$ is the period of $x$. Let us assume that the eigenvalues of $D_{x} f$ are positive. We prove the result when $\Sigma$ is an unstable separatrix, the other cases being analogous. As $\ell(\Sigma)<\infty$, the $\operatorname{limit}_{n \rightarrow \infty} f^{n}(y)$ exists. Let $z$ denote this limit, then $z \in \Delta$. Let $\sigma$ and $\tau$ be the eigenvalues of $D_{z} f$, with $|\sigma| \leq|\tau|$. Note that $|\sigma|<|\tau|$ since $z \in \Delta$ and $|\sigma| \leq 1$.

To start, assume that $y$ belongs to any of invariant manifolds of $z$. If $y \in W^{+}(z)$, see Figure 2-A, then $|\sigma|<1$ and consider two cases: (1) $|\tau|<1$ and $(2)|\tau|=1$. In the first case $z$ is a sink and the result follows. In the second case there exists a strong contractive foliation in a neighborhood of $z$, so the dynamics near to $z$ is decided by the dynamics on $W^{+}(z)$. As $y$ belongs to a stable separatrix of $z$ we are done.

Now assume that $y \in W^{-}(z)$. Observe that at $y, \Sigma$ and $W^{-}(z)$ meet transversally, because if the intersection were non transversal then $T_{x} W^{+}(x)=F(x)$, $T_{z} W^{-}(z)=E(z)$ and $y \in W^{+}(x) \cap W^{-}(z) \cap \Delta$, see Figure 2-B, would imply that $E(y)=F(y)$, which is an absurd. Consider the following three cases: (1) $W^{+}(z)$ has an unstable separatrix, $(2) W^{+}(z)$ has no two separatrices and (3) both separatrices of $W^{+}(z)$ are stable. In the first case we have $\ell(\Sigma)=\infty$, which follows from the $\lambda$-lemma when $|\tau|>1$, and from the existence of a strong contractive 
foliation when $|\tau|=1$, see Figure 2 $\mathrm{C}$. In the second case we get also the same conclusion, observe that here $|\tau|=1$ and $z$ is accumulated by fixed points in $W^{+}(z)$, see Figure 2-D. In the third case the result follows easily as seen in Figure 2-E.

If $y$ does not belong to $W^{-}(z) \cup W^{+}(z)$, then using analogous arguments as above we get the existence of $U$, see Figure 2 F.

If any of the eigenvalues of $D_{x} f$ is negative, the proof runs as above, but in this case it is possible that $z$ could be a period point of period two.

\section{Theorem's Proof}

Let $\Lambda$ be a basic set, $\left\{\gamma_{n}\right\}$ be an almost homoclinic sequence associated to $\Lambda$ and $p \in \Lambda$ be a periodic point. To simplify, we suppose that $p$ is a fixed point. Let $U$, $q^{s}, q^{u},\left\{q_{n}^{\prime}\right\}$ and $\left\{m_{n}^{\prime}\right\}$ as in the definition of an almost homoclinic sequence. We can assume that $\left\{\gamma_{n}\right\}=\left\{q_{n}^{\prime}, \ldots, f^{m_{n}^{\prime}}\left(q_{n}^{\prime}\right)\right\}$.

Let us see that we get the result when $W^{s}(\Lambda) \cap W^{u}(\Lambda) \backslash \Lambda \neq \emptyset$. Let $x^{s}, x^{u} \in \Lambda$ such that $W^{s}\left(x^{s}\right) \cap W^{u}\left(x^{u}\right) \neq \emptyset$. If there is a transversal meeting point between $W^{s}\left(x^{s}\right)$ y $W^{u}\left(x^{u}\right)$, then the result follows from the density of the manifolds $W^{s}(p)$ and $W^{u}(p)$ in $\Lambda$. If $W^{s}\left(x^{s}\right)$ and $W^{u}\left(x^{u}\right)$ does not intersects transversally then, using similar arguments to the Theorem 1's proof of [PT, pag. 36], we get that $f$ can be approximated in the $\mathrm{C}^{1}$ topology by a diffeomorphism which has a homoclinic tangency associated to $p$.

In what follows, we assume that $W^{s}(\Lambda) \cap W^{u}(\Lambda) \backslash \Lambda=\emptyset$. Let $\left\{f_{n}\right\},\left\{z_{n}\right\},\left\{q_{n}\right\}$, $\left\{m_{n}\right\}$ and $\left\{k_{n}\right\}$ as in lemma 1, and $\Delta_{n}=\left\{f_{n}^{k}\left(z_{n}\right): k \in \mathbb{Z}\right\} \cup\{p\}$. It is clear that $\Delta_{n}$ is a hyperbolic set for $f_{n}$, so it has a dominated splitting $E_{n} \oplus F_{n}$. By the same argument that in remark 1. $m_{n} \rightarrow \infty$ and $k_{n} \rightarrow \infty$.

Given $n \in \mathbb{Z}^{+}$we denote by $l_{n}$ the least positive integer such that

$$
\left\|\left.D f_{n}^{k}\right|_{E_{n}(x)}\right\|\left\|\left.D f_{n}^{-k}\right|_{F_{n}\left(f_{n}^{k}(x)\right)}\right\| \leq \frac{1}{2}
$$

for all $k \geq l_{n}$ and $x \in \Delta_{n}$. Since $\Delta_{n}$ has a dominated splitting, $l_{n}$ is well defined. The proof follows from the consideration of two cases: 1) $\liminf _{n \rightarrow \infty} l_{n}=\infty$, and 2) $\liminf _{n \rightarrow \infty} l_{n}=l<\infty$. Taking subsequences, if necessary, we can suppose that $l_{n} \rightarrow \infty\left(\right.$ resp. $\left.l_{n}=l\right)$ in the first (resp. second ) case. The next lemma finish the proof in the first case.

Lemma 6. If $l_{n} \rightarrow \infty$ then there exists a sequence $\left\{g_{n}\right\} \subset \operatorname{Diff}^{1}(M)$ such that $g_{n} \rightarrow f$ in the $\mathrm{C}^{1}$ topology, $p$ is a hyperbolic fixed point of $g_{n}$ and $g_{n}$ has a homoclinic tangency associated to $p$.

Proof. It is enough to show that, given $\theta>0$ and $\mathcal{U}$ neighborhood of $f$, there exists $g \in \mathcal{U}$ such that $p$ is a hyperbolic fixed point of $g$ and there is a homoclinic point of $g, x$ associated to $p$, such that the angle between $T_{x} W^{s}(p)$ and $T_{x} W^{u}(p)$ is less than $\theta$. Let $\theta>0$ and $\mathcal{U}$ a neighborhood of $f$ in $\operatorname{Diff}^{1}(M)$.

Let $N \in \mathbb{Z}^{+}$such that $f_{n} \in \mathcal{U}$, for all $n \geq N$. If there exist $x_{n} \in \Delta_{n}$ such that $\liminf _{n \rightarrow \infty} \angle\left(E_{n}\left(x_{n}\right), F_{n}\left(x_{n}\right)\right)<\theta$ then we have that we want. So assume that $\angle\left(E_{n}(x), F_{n}(x)\right) \geq \theta$ for all $x \in \Delta_{n}$ and $n \geq N$.

By hypothesis, there exist $x_{n} \in \Delta_{n}$ and $r_{n} \in \mathbb{Z}^{+}$, for each $n \in \mathbb{Z}^{+}$, such that $r_{n} \rightarrow \infty$ and

$$
\left\|\left.\left.D f_{n}^{r_{n}}\right|_{E_{n}\left(x_{n}\right)}|||| D f_{n}^{-r_{n}}\right|_{F_{n}\left(f_{n}^{r_{n}}\left(x_{n}\right)\right)}\right\|>\frac{1}{2}
$$


It is clear that $x_{n} \neq p$ for all $n$. Let $u_{n}^{k} \in E_{n}\left(f_{n}^{k}\left(x_{n}\right)\right)$ and $v_{n}^{k} \in F_{n}\left(f_{n}^{k}\left(x_{n}\right)\right)$, with $\left\|u_{n}^{k}\right\|=\left\|v_{n}^{k}\right\|=1$, for all $k, n \in \mathbb{Z}^{+}$. Let $B_{n}(k)$ be the matrix of $D f_{n}\left(f_{n}^{k}\left(x_{n}\right)\right)$ in the base $\left\{u_{n}^{k}, v_{n}^{k}\right\}$. Observe that $B_{n}(k)$ is a diagonal matrix and there exists $K>0$ such that $\left\|B_{n}(k)\right\| \leq K$, for all $k, n \in \mathbb{Z}^{+}$. Let $\epsilon>0$, given by Lemma 2 for $f$ and $\mathcal{U}$; $r_{0} \in \mathbb{Z}^{+}$, given by Lemma 1 for $\epsilon, \theta$ and $K$, and $n \geq N$ such that $r_{n} \geq r_{0}$. We define $A_{k}=B_{n}(k)$, for $k \geq 0$. Observe that $\left\{A_{k}\right\}_{k=0}^{r_{n}}$ satisfy the hypothesis of Lemma $\mathbb{E}$, so there are matrices $\tilde{A}_{0}, \ldots, \tilde{A}_{r_{n}-1}$ such that $\left\|\tilde{A}_{k}-A_{k}\right\|<\epsilon, \tilde{A}_{k} e_{1}=e_{1}$ and the angle between $e_{1}$ and $\tilde{A}_{r_{n}-1} \circ \cdots \circ \tilde{A}_{0} e_{2}$ is less than $\theta$. By Lemma 2, there exits $g \in \mathcal{U}$ such that $g^{j}\left(x_{n}\right)=f_{n}^{j}\left(x_{n}\right)$, for all $k \in \mathbb{Z}$ and the angle between $T_{y} W^{s}(p)$ and $T_{y} W^{u}(p)$ is less than $\theta$, with $y=f_{n}^{r_{n}}\left(x_{n}\right)$, that is what we wanted to proof.

Lets go consider, to finish, the second case, i.e. when $l_{n}=l$. We will see that this case drives us to a contradiction if we assume that, as in fact we are doing, that $W^{s}(\Lambda) \cap W^{u}(\Lambda) \backslash \Lambda=\emptyset$.

There exist a sequence $\left\{n_{i}\right\}$ such that $\left\{\Delta_{n_{i}}\right\}$ converges, in the Hausdorff topology, to a compact set $\Delta$. To simplify suppose that $n_{i}=i$. Since $l_{n}=l$, then $\Delta$ has a dominated splitting $E \oplus F$. The subbundles $E$ and $F$ can be extended in a continuous way to a neighborhood $V_{0}$ of $\Delta$ in such a way that $E(x) \oplus F(x)=T_{x} M$, for all $x \in V_{0}$. Given $\eta \in T_{x} M$, for $x \in V_{0}$, we denote by $\eta_{1} \in E(x)$ and $\eta_{2} \in F(x)$ to the vectors such that $\eta=\eta_{1}+\eta_{2}$. Let

$$
C_{r}^{E}(x)=\left\{\eta \in T_{x} M:\left\|\eta_{2}\right\|<r\left\|\eta_{1}\right\|\right\} \quad \text { y } \quad C_{r}^{F}(x)=\left\{\eta \in T_{x} M:\left\|\eta_{1}\right\|<r\left\|\eta_{2}\right\|\right\},
$$

for $r>0$.

Let $V_{1}$ be an open neighborhood of $\Delta, 0<\rho<1$ and $\frac{1}{2}<\lambda<1$ be constants such that $\overline{V_{1}} \subset V_{0}$,

$$
\begin{aligned}
& D f^{-1} C_{\rho}^{E}(x) \subset C_{\rho}^{E}\left(f^{-1}(x)\right), \quad D f C_{\rho}^{F}(x) \subset C_{\rho}^{F}(f(x)) \quad \forall x \in \overline{V_{1}} \\
& \left\|\left.D f^{k}\right|_{C_{\rho}^{E}(x)}\right\|\left\|\left.D f^{-k}\right|_{C_{\rho}^{F}\left(f^{k}(x)\right)}\right\|<\lambda \quad \forall k \geq l \forall x \in \bigcap_{j=0}^{k} f^{-j}\left(\overline{V_{1}}\right) .
\end{aligned}
$$

Let $\Delta_{0}^{ \pm}=\bigcap_{j=0}^{\infty} f^{ \pm j}\left(\overline{V_{1}}\right)$ and $\Delta_{0}=\Delta_{0}^{+} \cap \Delta_{0}^{-}$. We observe that $\Delta \subset \Delta_{0}, f\left(\Delta_{0}^{+}\right) \subset$ $\Delta_{0}^{+}$and $f^{-1}\left(\Delta_{0}^{-}\right) \subset \Delta_{0}^{-}$.

We call an interval to the image of an immersion in $M$ of a closed interval and we will denote by $\ell(I)$ its length. We shall say that an interval $I$ is an E-interval if $I \subset \overline{V_{1}}$ and $T_{x} I \subset C_{\rho}^{F}(x)$, for all $x \in I$. Analogously, we define $F$-intervals. An $E$-interval $I$ is a $\delta$-E-interval if $I \subset \Delta_{0}^{+}$and $\ell\left(f^{k}(I)\right)<\delta$, for all $k \geq 0$. In the same way, we say that an $F$-interval $I$ is a $\delta$-F-interval if $I \subset \Delta_{0}^{-}$and $\ell\left(f^{-k}(I)\right)<\delta$, for all $k \geq 0$.

Now we state Proposition 3.1 of $[$ PS].

Proposition 1. There exists $\delta_{0}>0$ such that if $I$ is a $\delta$-E-interval, with $0<\delta \leq$ $\delta_{0}$, then one of the following alternatives hold:

i. $\omega(I)=\bigcup_{k=1}^{m} \mathcal{C}_{k}$, where each $\mathcal{C}_{k}$ is a simple closed curve, normally hyperbolic for $f^{m}$, with its dynamics an irrational rotation and $f\left(\mathcal{C}_{k-1}\right)=\mathcal{C}_{k}$, for $1 \leq k \leq m$, with $\mathcal{C}_{0}=\mathcal{C}_{m}$.

ii. $\omega(I)$ has just periodic points.

We recall that there are points $q_{n}$ such that $q_{n} \rightarrow q^{u}$ and $f^{k_{n}}\left(q_{n}\right) \rightarrow q^{s}$, see Figure 11. We observe that $q^{s}, q^{u} \in \Delta$, because of $q^{s}=\lim _{n \rightarrow \infty} f_{n}^{m_{n}}\left(z_{n}\right), q^{u}=$ $\lim _{n \rightarrow \infty} z_{n}$ and $z_{n} \in \Delta_{n}$, for all $n$. Let $V_{2}$ be an open neighborhood of $\Delta, \delta_{1}>0$ 
and $N_{0} \in \mathbb{Z}^{+}$be such that $\overline{V_{2}} \subset V_{1}, B_{\delta_{1}}\left(V_{2}\right) \subset V_{1}$ and $\Delta_{n} \subset V_{2}$, for $n \geq N_{0}$. The item (v) of Lemma 1 implies that we there exists $N_{1} \geq N_{0}$ such that $f^{j}\left(q_{n}\right) \in V_{2}$, for $0 \leq j \leq k_{n}$, if $n \geq N_{1}$.

Lemma 7. For all $\epsilon>0$ there exists $n \in \mathbb{Z}^{+}$such that the following holds:

i. $\operatorname{dist}\left(q_{n}, q^{u}\right)<\epsilon$.

ii. If $I$ is an E-interval with $q_{n}$ in the interior of $I$ and the length of one of each connected components of $I \backslash\left\{q_{n}\right\}$ is greater than $\epsilon$ then there exists $J \subset I$, E-interval, such that $q_{n} \in \partial J, \ell\left(f^{j}(J)\right) \leq \epsilon$, for $0 \leq j \leq k_{n}$, and the extreme point of $f^{k_{n}}(J)$ which is not $f^{k_{n}}\left(q_{n}\right)$ belongs to the connected component of $B_{\epsilon}\left(q^{s}\right) \cap W^{s}\left(p^{s}\right)$ that contains $q^{s}$.

Proof. Let $\psi:[-1,1]^{2} \rightarrow M$ be $\mathrm{C}^{1}$ coordinates such that $\psi(0,0)=q^{s} ; \eta \in C_{\rho}^{E}(x)$ (resp. $\left.\eta \in C_{\rho}^{F}(x)\right)$ is written as $(u, v)$ in such coordinates, with $\|v\|<\|u\|$ (resp. $\|u\|<\|v\|) ; B=\psi\left([-1,1]^{2}\right) \subset V_{2}$, and $\Gamma=\psi([-1,1] \times\{0\})$ is the connected component of $W^{s}\left(p^{s}\right) \cap B$ which contains $q^{s}$. There exists $N_{2} \geq N_{1}$ such that $f^{k_{n}}\left(q_{n}\right) \in \psi\left([-1 / 2,1 / 2]^{2}\right)$, for all $n \geq N_{2}$. If $I$ is an $E$-interval with $q_{n} \in \partial I$ we shall say that it point to $\Gamma$, if either there is $J \subset I$, an $E$-interval with $q_{n} \in J$ and $f^{k_{n}}(J) \subset B$, such that the second coordinate of every point in $f^{k_{n}}(J)$ is less than or equal to, in absolute value, the second coordinate of $f^{k_{n}}\left(q_{n}\right)$, or $f^{k_{n}}(J) \cap \Gamma \neq$ $\emptyset$. Let $\zeta_{n}=\sqrt{2}\left|y_{n}\right|$, for $n \geq N_{2}$, where $\psi\left(x_{n}, y_{n}\right)=f^{k_{n}}\left(q_{n}\right)$. We observe that $\lim _{n \rightarrow \infty} \zeta_{n}=0$, and if $J$ is an $E$-interval which points to $\Gamma$, with $q_{n} \in \partial J$, and $\ell\left(f^{k_{n}}(J)\right) \geq \zeta_{n}$ then $f^{k_{n}}(J)$ meets $\Gamma$.

Let $0<\epsilon<\min \left(\delta_{0}, \delta_{1}\right)$ be fixed and $N_{3} \geq N_{2}$ such that $q_{n} \in B_{\epsilon}\left(q^{u}\right)$, for all $n \geq N_{3}$, where $\delta_{0}$ is given by Proposition 1. For each $n \geq N_{3}$, let $\tilde{I}_{n}$ be an $E$-interval that satisfies the hypothesis of the lemma, i.e. $\tilde{I}_{n} \backslash\left\{q_{n}\right\}$ has two connected components with its length greater than $\epsilon$. Let $I_{n}$ be the unique $E$ interval contained in $\tilde{I}_{n}$ which point to $\Gamma$ and has lengths $\epsilon$. We observe that by definition $q_{n} \in \partial I_{n}$. Let $I_{n}^{0}, \ldots, I_{n}^{k_{n}}$ be the $E$-intervals which satisfy: $I_{n}^{0} \subset I_{n}$, $q_{n} \in \partial I_{n}^{0}, f^{j}\left(I_{n}^{0}\right)=I_{n}^{j}$ and $\epsilon=\max \left\{\ell\left(I_{n}^{j}\right): 0 \leq j \leq k_{n}\right\}$. We note that $\left\{I_{n}^{j}\right\}$ is well defined since $\ell\left(I_{n}\right)=\epsilon$.

If we prove that there is $n \geq N_{3}$ such that $I_{n}^{k_{n}}$ intersects $\Gamma$ then we will have proved the lemma. Let us prove the existence of such an $n$ by contradiction. So we assume that $I_{n}^{k_{n}} \cap \Gamma=\emptyset$, for all $n \geq N_{3}$, and get an absurd.

Let $\nu_{n}=\max \left\{0 \leq j \leq k_{n}: \ell\left(I_{n}^{j}\right)=\epsilon\right\}$. Note that $k_{n}-\nu_{n} \rightarrow \infty$, if $n \rightarrow \infty$, since $\zeta_{n} \rightarrow 0$. Let $J_{n}=I_{n}^{k_{n}}$, by compactness there exists a subsequence $J_{n_{j}}$ which converges to an $E$-interval $J$ in such a way that $x=\lim _{j \rightarrow \infty} f^{\nu_{n_{j}}}\left(q_{n_{j}}\right)$ is well defined. It is clear that $\ell(J)=\epsilon$ and $x \in \partial J$. Without loss of generality we assume that $J_{n} \rightarrow J$ and $f^{\nu_{n}}\left(q_{n}\right) \rightarrow x$.

$J$ is an $\epsilon$-E-interval (because of $k_{n}-\nu_{n} \rightarrow \infty$ ) and $x \in \Delta$, see (N) of lemma 1 . Proposition 1 implies that either $\omega(J)$ is a finite collection of invariant curves normally hyperbolic or $\omega(J)$ is formed by periodic points. Let us see that each alternatives lead us to a contradiction. We observe that $\omega(J) \subset \Delta_{0}$.

Consider the case when $\omega(J)$ is a finite collection of invariant curves normally hyperbolic. Since the inner dynamics of theses curves are irrational and $F$ restricted to theses curves is its tangent space, the continuity of the dominated splitting implies that $E$ is a normal fiber which is contractive. So $\omega(J)$ is an attractor, i.e. $W^{s}(\omega(J))$ is a neighborhood of $\omega(J)$. Since $x \in W^{s}(\omega(J)), q_{n} \in W^{s}(\omega(J))$ for $n$ 


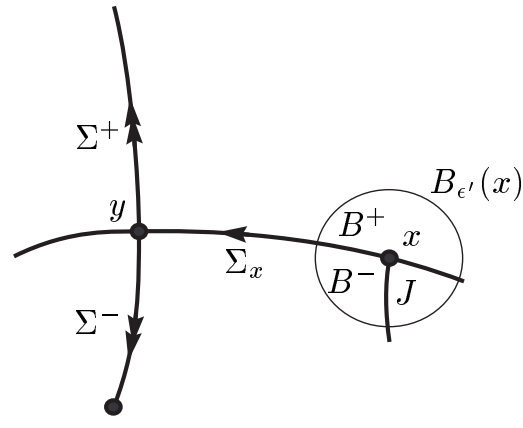

A

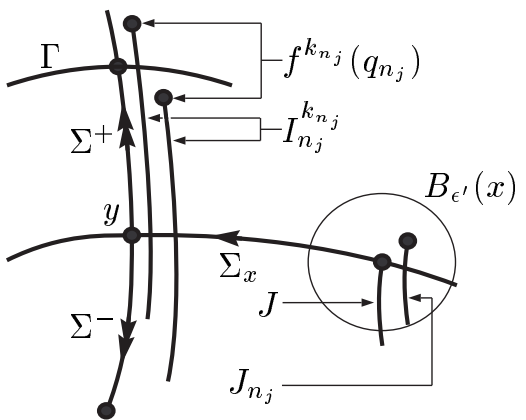

B

Figure 3. Proof of Lemma 目

big enough, but this is impossible, because $\nu_{n}<k_{n}, f^{k_{n}}\left(q_{n}\right) \rightarrow q^{s} \in W^{s}(p)$ and $p \notin \omega(J)$.

Now consider the case when $\omega(J)$ is formed just by periodic points. Let $y \in \omega(x)$, $\sigma$ and $\tau$ be the eigenvalues associated to $y$, with $|\sigma|<|\tau|, W^{-}(y)$ and $W^{+}(y)$ be the invariant manifolds associated to $\sigma$ and $\tau$ respectively. We observe that $|\sigma| \leq 1$. With no loss of generality we suppose that $y$ is a fixed point and $\sigma, \tau \in \mathbb{R}^{+}$. We have to consider several subcases.

$x$ belongs to the interior of $W^{s}(y)$ : In this subcase we get an absurd in an analogous way as when we consider $\omega(J)$ as a finite collection of invariant curves normally hyperbolic.

$x$ does not belong to the interior of $W^{s}(y)$ and $x=y$ : The proof here is similar to the proof of the next subcase.

$x$ does not belong to the interior of $W^{s}(y)$ and $x \neq y$ : Then $\tau \geq 1$ and $x \in$ $W^{-}(y)$. We get that $W^{+}(y)$ has at most one stable separatrix. Denote by $\Sigma_{x}$ the separatrix of $W^{-}(y)$ which contains $x$. We are going to obtain a contradiction.

Assume that $\tau>1$. Let $\epsilon^{\prime}>0$ be small and $\Sigma_{x}^{0}$ be the connected component of $B_{\epsilon^{\prime}}(x) \cap \Sigma_{x}$ which contains $x . B_{\epsilon^{\prime}}(x) \backslash \Sigma_{x}^{0}$ has two connected components, we denote them by $B^{-}$and $B^{+}$. $J$ intercepts just one of them, let us say $B^{-}$. The $\lambda$ lemma $(\sqrt{\mathrm{P}})$ implies that $\left\{f^{k}(J)\right\}$ accumulates in one of the separatrices of $W^{+}(y)$, see Figure 3 -A. Let $\Sigma^{-}$be this separatrix and $\Sigma^{+}$be the other one of $W^{+}(y)$. Since $\left\{f^{k}(J)\right\}$ accumulates on $\Sigma^{-}, \ell\left(\Sigma^{-}\right) \leq \epsilon$.

Claim 1. If there are infinitely many $n$ for which $f^{\nu_{n}}\left(q_{n}\right) \in B^{+}$then $\ell\left(\Sigma^{+}\right) \leq \epsilon$.

Let us prove the claim by contradiction. We have that $q^{s} \notin \Sigma_{x}$. Let $\left\{n_{j}\right\}$ be an increasing sequence of positive integers such that $f^{\nu_{n_{j}}}\left(q_{n_{j}}\right) \in B^{+}$, for all $j$, and $n_{1} \geq N_{3}$. Since $\ell\left(\Sigma^{+}\right)>\epsilon$, there is $r_{j} \in \mathbb{Z}^{+}$such that $\ell\left(f^{r_{j}}\left(J_{n_{j}}\right)\right)>\epsilon$. From here it follows that $\nu_{n_{j}}+r_{j} \geq k_{n_{j}}$ and $q^{s} \in \Sigma^{+}$. Given $j$ big enough we have two alternatives. Either $I_{n_{j}}$ does not point to $\Gamma$ or $I_{n_{j}}^{k_{n_{j}}}$ intersects $\Gamma$, see Figure 3-B. For both of the alternatives this is an absurd. This ends the proof of the Claim 1.

Suppose now that there is an increasing sequence of positive integers $\left\{n_{j}\right\}$ such that $f^{\nu_{n_{j}}}\left(q_{n_{j}}\right) \in B^{+}$, for all $j \geq 1$, then there exist $z \in \Sigma^{+}$and $\left\{r_{j}\right\}$, with $\nu_{n_{j}}<r_{j}<k_{n_{j}}$, such that $f^{r_{j}}\left(q_{n_{j}}\right) \rightarrow z$. From here it follows that $z \in \Delta$. Lemma 5 give us the existence of a periodic point $z^{\prime}$ and a small neighborhood $V$ of $z$ which 
is contained in the stable manifold of the orbit of $z^{\prime}$, which is an absurd. We remark that the same conclusion is obtained if there is $\left\{n_{j}\right\}$ as above such that $f^{\nu_{n_{j}}}\left(q_{n_{j}}\right) \in B^{-}$. So we have that $f^{\nu_{n}}\left(q_{n}\right) \in \Sigma_{x}$, for $n$ big enough, again an absurd, because $\Sigma_{x} \subset W^{s}(y)$. With this we get the third subcase when $\tau>1$.

If $\tau=1$ then again there are several alternatives. $W^{+}(y)$ can have no separatrices, two or one; moreover if it has one separatrix this can be an stable or an unstable separatrix. For each of this cases we get an absurd in the same way as above. This finishes the proof of the lemma.

An immediate consequence of the lemma is the following result.

Corollary 2. If $\left\{\tilde{I}_{n}\right\}$ is a sequence of E-intervals such that $q_{n} \in \tilde{I}_{n}$ and each of the connected components of $\tilde{I}_{n} \backslash\left\{q_{n}\right\}$ has length $\delta_{2}=\min \left\{\delta_{0}, \delta_{1}\right\} / 2$ then there are sequences $\left\{n_{j}\right\} \subset \mathbb{Z}^{+}$and $\left\{J_{j}\right\}$ such that

i. $n_{j} \rightarrow \infty$

ii. $J_{j}$ is an E-interval with $q_{n_{j}} \in \partial J_{j}$ and $\ell\left(f^{k}\left(J_{j}\right)\right) \leq 1 / j$, for $0 \leq k \leq k_{n_{j}}$

iii. $\partial f^{k_{n_{j}}}\left(J_{j}\right)=\left\{f^{k_{n_{j}}}\left(q_{n_{j}}\right), x_{j}\right\}$, with $x_{j} \in \Gamma$, where $\Gamma$ is defined as in the lemma's proof.

Lemma 7 has a version for $F$-intervals, in such a version $f^{k_{n}}\left(q_{n}\right)$ is changed by $q_{n}$ and $f^{-1}$ by $f$. We remark that the properties of $\left\{q_{n}\right\}$ used in the proof are: (1) $\bigcup_{j=0}^{k_{n}} B_{\epsilon}\left(f^{j}\left(q_{n}\right)\right) \subset \overline{V_{1}}$, for $\epsilon$ small and $n$ big, and $(2) \lim _{n \rightarrow \infty} q_{n}=q^{u}$ and $\lim _{n \rightarrow \infty} f^{k_{n}}\left(q_{n}\right)=q^{s}$. On the other hand, $\left\{x_{j}\right\}$, given by Corollary 2, satisfies: (1) $\bigcup_{k=0}^{k_{n_{j}}} B_{\epsilon}\left(f^{-k}\left(x_{j}\right)\right) \subset \overline{V_{1}}$, if $\epsilon$ is small and $j$ big, and (2) $\lim _{j \rightarrow \infty} x_{j}=q^{s}$ and $\lim _{j \rightarrow \infty} f^{-k_{n_{j}}}\left(x_{j}\right)=q^{u}$. So a version for $F$-intervals is true changing the sequence $\left\{q_{n}\right\}$ by the sequence $\left\{x_{j}\right\}$. In particular, if we take as $F$-interval to $\Gamma$ we get that $W^{s}\left(p^{s}\right)$ intercepts transversally to $W^{u}\left(p^{u}\right)$ near of $q^{u}$, so $W^{s}(\Lambda) \cap W^{u}(\Lambda) \backslash \Lambda \neq \emptyset$, which is a contradiction, since we are assuming that $W^{s}(\Lambda) \cap W^{u}(\Lambda) \backslash \Lambda=\emptyset$. So we conclude the proof of the theorem.

\section{REFERENCES}

[D] Díaz LJ: Robust nonhyperbolic dynamics and heterodimensional cycles. Ergodic Theory and Dynamical Systems 15(1995) no. 2, 291-315.

[F] Franks J: Necessary conditions for stability of diffeomorphisms. Trans. AMS 158(1971), 301-308.

[H] Hayashi S: Connecting invariant manifolds and the solution of the $C^{1}$-stability and $\Omega$ stability conjectures for flows. Ann. of Math. 145(1997), 81-137.

[P] Palis J: On Morse-Smale dynamical systems. Topology 8(1969) 385-405.

$[\mathrm{PT}]$ Palis J and Takens F: Hyperbolicity 85 sensitive chaotic dynamics at homoclinic bifurcations Cambridge University Press, 1993.

[PS] Pujals E and Sambarino M: Homoclinic tangencies and hyperbolicity for surfaces diffeomorphisms. Ann. of Math. (2) 151(2000)no.3, 961-1023.

[WX] Wen L and Xia Z: $\mathrm{C}^{1}$ connecting lemmas. Transactions of the A.M.S.352(2000)no. 11, $5213-5230$.

Departamento de Matemáticas Puras y Aplicadas, Universidad Simón Bolívar, Apartado Postal 89000, Caracas 1086-A, Venezuela

E-mail address: jmartin@usb.ve

Departamento de Matemática, Facultad de Ciencias, la Hechicera, Ula, Mérida 5101, Venezuela

E-mail address: lmora@ula.ve 